\title{
In vitro evaluation of the impact of human background microbiota on the response to Bifidobacterium strains and fructo-oligosaccharides
}

\author{
Silvia Arboleya ${ }^{1}$, Nuria Salazar ${ }^{1}$, Gonzalo Solís ${ }^{2}$, Nuria Fernández ${ }^{3}$, Miguel Gueimonde ${ }^{1}$ \\ and Clara G. de los Reyes-Gavilán ${ }^{1 *}$ \\ ${ }^{1}$ Department of Microbiology and Biochemistry of Dairy Products, Instituto de Productos Lácteos de Asturias, \\ Consejo Superior de Investigaciones Científicas (IPLA-CSIC), Paseo Rí Linares s/n, 33300 Villaviciosa, Asturias, Spain \\ ${ }^{2}$ Paediatrics Service, Hospital Universitario Central de Asturias, SESPA, Oviedo, Asturias, Spain \\ ${ }^{3}$ Paediatrics Service, Hospital de Cabueñes, SESPA, Gijón, Asturias, Spain
}

(Submitted 21 November 2012 - Final revision received 3 April 2013 - Accepted 11 April 2013 - First published online 31 May 2013)

\begin{abstract}
The microbial colonisation of the infant gut begins immediately after birth and is essential for the development of the intestine, the immune system and later well-being. Important differences have been reported in the characteristics of such microbiota in different infant population groups. In the present study, we employed an in vitro faecal batch culture model using faeces from different human population groups (adults and full-term breast-fed, full-term formula-fed and preterm infants) to determine the influence that the addition of four bifidobacterial strains and fructo-oligosaccharides (FOS) exerts on the profile of SCFA measured by GC as well as on the levels of some relevant intestinal microbial groups by quantitative PCR during incubation. Differences were found in the levels of SCFA and intestinal microbial groups in the faecal cultures depending on the human group origin of the faecal samples $(P<0 \cdot 05)$, this being a predominant factor, compared with bifidobacteria or FOS added, in determining microbiota dynamics. These results exhibit the importance of the initial characteristics of the basal intestinal microbiota in the effect exerted by bifidobacteria or FOS that are added and highlight the need to design probiotics targeting specific human population groups.
\end{abstract}

Key words: Bifidobacterium: Intestinal microbiota: Infant population groups: Probiotics

The microbial colonisation of the infant gut begins immediately after birth with facultative anaerobes, such as enterobacteria, enterococci and lactobacilli, followed by the establishment of anaerobic genera, including Bifidobacterium, Bacteroides and Clostridium. Some factors such as the mode of delivery, feeding habits, use of medication or gestational age may affect this process ${ }^{(1)}$. Important differences have been found in the process of establishment of the intestinal microbiota and in the characteristics of such microbiota in full-term breast-fed, full-term formula-fed and premature babies. Fullterm breast-fed infants are considered as the 'gold standard' of a healthy intestinal microbiota for neonates. This group of newborns develops an intestinal microbiota rich in bifidobacteria and displays differences in metabolomic profiles with respect to formula-fed babies ${ }^{(2-4)}$. Preterm infants have altered facultative anaerobe to strict anaerobe ratios and lower levels of SCFA than full-term breast-fed infants ${ }^{(5,6)}$. After weaning, the levels of bifidobacteria in the faeces of breast-fed infants do not change, but an increase of some other microbial groups, such as enterobacteria and enterococci, seems to occur ${ }^{(7)}$.

Probiotics are defined as live microorganisms which when administered in adequate amounts confer a benefit on the host $^{(8)}$. Probiotics that are most frequently consumed by humans belong to the genera Lactobacillus and Bifidobacterium. The use of probiotics and/or prebiotics to restore or maintain the adequate composition and metabolic activity of the intestinal microbiota has been proposed, and some beneficial effects of specific probiotic strains have been reported ${ }^{(9)}$. The initial stages of intestinal microbiota development seem to be the appropriate ones for microbiota modulation ${ }^{(6,10)}$. However, the outcome of the probiotic intervention may vary depending on different factors, such as the strain used or the target population, among others ${ }^{(11-13)}$. In this regard, a recent human intestinal mucosal transcriptome study assessing the effect of the administration of different probiotics has shown that changes in gene expression are more dependent on the individual than on the probiotic strain used ${ }^{(14)}$. Another study has

Abbreviations: CSIC, Consejo Superior de Investigaciones Científicas; FOS, fructo-oligosaccharides.

*Corresponding author: Dr C. G. d. 1. Reyes-Gavilán, fax +349858922 33, email greyes_gavilan@ipla.csic.es 
highlighted that the different impacts that the administration of probiotics has on the gut microbiota of infants depend on the mode of feeding and initial microbiota composition ${ }^{(15)}$.

The aim of the present study was to determine the impact that the addition of some bifidobacteria strains and fructooligosaccharides (FOS) exerts on the intestinal microbiota of different groups of newborns (breast-fed, formula-fed and premature babies) and adults by using an in vitro faecal batch culture model, specifically developed for infants in a previous work ${ }^{(16)}$.

\section{Materials and methods}

\section{Strains and growth conditions}

In the present study, four Bifidobacterium strains belonging to different species were used: Bifidobacterium animalis Bb12 (commercial origin) ${ }^{(17)}$; Bifidobacterium longum BM 18/6 (IPLA 20 002) and Bifidobacterium breve BM 13/14 (IPLA $20005)$, both from breast milk ${ }^{(18)}$; Bifidobacterium bifidum IF 10/20 (IPLA 20 025) (infant faecal origin) ${ }^{(16)}$. Micro-organisms were grown in de Man, Rogosa and Sharpe (MRS) medium (Difco, Becton Dickinson) supplemented with 0.25\% (w/v) L-cysteine (Sigma Chemical Company) and were incubated at $37^{\circ} \mathrm{C}$ in an anaerobic chamber (Mac 500; Don Whitley Scientific) under a $10 \%(\mathrm{v} / \mathrm{v}) \mathrm{H}_{2}, 10 \%(\mathrm{v} / \mathrm{v}) \mathrm{CO}_{2}$ and $80 \%(\mathrm{v} / \mathrm{v}) \mathrm{N}_{2}$ atmosphere. Before the start of the experiments, strain identity was confirmed by partial amplification of the $16 \mathrm{~S}$ ribosomal RNA gene using primers $\mathrm{Y} 1-\mathrm{Y} 2^{(19)}$.

\section{Volunteers and faecal sample preparation}

Faecal samples were obtained from four human populations: preterm neonates; full-term formula-fed infants; full-term breast-fed babies; adults. Faeces from eighteen infants were collected at the Neonatology Units of the Asturias Central Hospital (Oviedo, Asturias, Spain) and Cabueñes Hospital (Gijón, Asturias, Spain), and adults' faeces were provided by three healthy donors (between 26 and 44 years old) who were on an omnivorous unrestricted diet and had not taken antibiotics during the 2 months leading up to the study. The present study was conducted according to the guidelines laid down in the Declaration of Helsinki, and all procedures involving human subjects were approved by the Regional Ethical Committee of Asturias Public Health Service (SESPA) and by the Bioethics Subcommittee of Consejo Superior de Investigaciones Científicas (CSIC). Written informed consent was obtained from the mothers of infants and from adult donors.

Fresh faecal samples were immediately placed in anaerobic jars (Anaerocult A System; Merck), transported to the laboratory and processed within $2 \mathrm{~h}$ of collection. All infants were vaginally delivered. The samples were collected from 2- to 3-week-old full-term breast-fed and premature babies (gestational age 32-34 weeks). The birth weight interval of preterm infants varied approximately from 1600 to $2300 \mathrm{~g}$ and that of breast-fed infants ranged from 3200 to $4300 \mathrm{~g}$. The birth weight of full-term formula-fed infants ranged from 2700 to $4400 \mathrm{~g}$, and faeces were collected from 5- to 6-week-old infants.

Faecal samples that were available were mixed in equal proportions, diluted $1: 10(\mathrm{w} / \mathrm{v})$ in a pre-reduced PBS solution and homogenised in a Lab Blender 400 Stomacher (Seward Medical) for $5 \mathrm{~min}$. The homogenised faecal mixtures were added to a basal medium for cultivation of infant faeces (BMIF) $(10 \%, \mathrm{v} / \mathrm{v})^{(16)}$ and were stabilised for $4 \mathrm{~h}$ under anaerobic conditions at $37^{\circ} \mathrm{C}$.

\section{Faecal batch culture conditions}

For each human population group, three independent trials were carried out. Faecal inocula were prepared at each time with a mixture of faeces from two to three different individuals for each population group in the case of infants and with a mixture of faeces from two of the three donors participating in the present study in the case of adults. To the stabilised faecal mixtures in BMIF was added 5\% (v/v) reconstituted formula milk. This medium has previously been proven to support a balanced growth of selected strains belonging to the main commensal and pathogenic microbial species inhabiting the infant gut ${ }^{(16)}$. For each batch and human group, the faecal mixtures in BMIF-milk were distributed into different tubes, into which the four bifidobacterial strains were inoculated at a final concentration of $10^{6}$ colony-forming units $/ \mathrm{ml}$. To another tube containing BMIF-milk was added FOS (Orafti P95; BENEO-Orafti) at a $0.3 \%(\mathrm{w} / \mathrm{v})$ final concentration, and one additional tube was kept without adding the bifidobacterial strains or FOS and was used as the negative control. Fermentations were carried out at $37^{\circ} \mathrm{C}$ under anaerobic conditions for $2 \mathrm{~d}$ and tubes were withdrawn at 0,5 and $48 \mathrm{~h}$. Cultures $(1 \mathrm{ml})$ were centrifuged (12000 $\boldsymbol{g}$ for $15 \mathrm{~min}$ ) each time, and pellets and supernatants were collected. Determination of $\mathrm{pH}$ at different times was done by direct measurement with a $\mathrm{pH}$ meter Basic $20+$ (Crison Instruments S.A.).

\section{Analysis of SCFA and lactate by GC-MS/flame injection detector and HPLC}

Supernatants were filtered through $0 \cdot 2 \mu \mathrm{m}$ filters and were used for the quantification of SCFA by GC and lactic acid by HPLC. A chromatographic system composed of two 6890N GC (Agilent Technologies, Inc.) connected to a flame injection detector and a MS 5973N detector (Agilent) was used for the quantification and identification of SCFA as described previously $^{(5)}$. Total SCFA (expressed in $\mathrm{mm}$ concentrations) were calculated as the sum of the three major SCFA (acetic, propionic and butyric acids). The molar proportion of each SCFA was obtained as the concentration percentage with respect to the total SCFA.

Lactic acid was quantified with a HPLC chromatographic system composed of an Alliance 2690 module injector, a PDA 996 photodiode array detector and the Empower software (Waters). Chromatographic conditions were set and calculations were carried out as described previously ${ }^{(20)}$, and lactic acid concentrations are expressed in $\mathrm{mm}$. 


\section{Analysis of faecal microbial populations by quantitative $P C R$}

DNA was extracted from the pellets and was used for the quantification of Enterobacteriaceae and Enterococcaceae families and Bifidobacterium, Bacteroides and Weissella groups by quantitative PCR by converting $16 \mathrm{~S}$ ribosomal DNA copies to cell counts. The number of cells was obtained by comparing the $C_{\mathrm{t}}$ values obtained from a standard curve constructed using the pure cultures of appropriate strains in which the $C_{\mathrm{t}}$ values were plotted as a linear function of the base-10 logarithm of the number of cells calculated by plate counting. Primers and conditions used were those described previously ${ }^{(5)}$.

\section{Statistical analysis}

Statistical analysis of the results was carried out using SPSS software version 15.0 (SPSS, Inc.). A one-way ANOVA test was carried out to determine the differences in SCFA and microbial population levels among faecal slurries from the different human population groups after stabilisation; post hoc comparison was achieved using a least significant difference test $(P<0.05)$. In the case of faecal cultures, a repeated-measures ANOVA test was performed, considering the time of incubation as the intra-subject factor and the human population group (preterm, breast-fed and formulafed infants and adults) and treatment (bifidobacteria or FOS added, and negative control) as inter-subject factors $(P<0 \cdot 05)$. Post hoc pairwise comparisons of parameters among the human groups considered in the present study (SCFA, lactic acid and microbial groups) were assessed by Bonferroni's test $(P<0 \cdot 05)$.

\section{Results \\ Composition of faecal slurries from the different population groups after stabilisation}

Faeces were stabilised at $37^{\circ} \mathrm{C}$ for $4 \mathrm{~h}$ in the BMIF medium before the preparation of faecal cultures, and variations in SCFA and lactate patterns, as well as in microbiota composition, were determined after stabilisation. Clear differences among faecal slurries from the different human population groups were evidenced (Table 1 ). Thus, significantly higher molar proportions $(P<0.05)$ of acetate and butyrate and lower proportions of propionate were found in the stabilised faecal slurries of breast-fed infants than in those of the other human groups. In contrast, the stabilised samples from premature babies contained the lowest proportions of butyrate and the highest levels of lactate. Regarding the microbial groups, the faecal slurries from premature babies displayed the lowest levels and those from adults exhibited the highest levels $(P<0.05)$ of Bacteroides, whereas Bifidobacterium was found to be more abundant in the stabilised samples from formula-fed infants than in those from the other groups. Significantly lower amounts of Enterococcaceae and Weissella were found in the samples from breast-fed infants than in those from the other groups, whereas, conversely, the stabilised faecal slurries from premature babies displayed the highest counts of Enterobacteriaceae and Weissella.

\section{Effect of the addition of bifidobacteria and fructo- oligosaccharides on SCFA, lactic acid and intestinal microbial group dynamics in faecal cultures from the different human population groups}

After the stabilisation process, faecal cultures with bifidobacteria or FOS, as well as the negative control without any

Table 1. Levels of SCFA and lactic acid and counts of different intestinal microbial groups determined by quantitative PCR in the slurries of three faecal mixtures corresponding to the different human population groups after a stabilisation period of $4 \mathrm{~h}$ during anaerobiosis at $37^{\circ} \mathrm{C}$

(Mean values with their standard errors)

\begin{tabular}{|c|c|c|c|c|c|c|c|c|}
\hline \multirow[b]{2}{*}{ Human groups } & \multicolumn{2}{|c|}{ Premature } & \multicolumn{2}{|c|}{ Formula-fed } & \multicolumn{2}{|c|}{ Breast-fed } & \multicolumn{2}{|c|}{ Adult } \\
\hline & Mean & SE & Mean & SE & Mean & SE & Mean & SE \\
\hline \multicolumn{9}{|c|}{ Molar proportions (\%)* } \\
\hline Acetate & $83.53^{a, b}$ & 0.65 & $82 \cdot 33^{a}$ & 0.63 & $84 \cdot 87^{\mathrm{b}}$ & 0.50 & $81.95^{\mathrm{a}}$ & 0.62 \\
\hline Propionate & $14 \cdot 62^{\mathrm{a}}$ & 0.54 & $14 \cdot 19^{a}$ & 0.50 & $8 \cdot 22^{b}$ & 0.27 & $15 \cdot 23^{a}$ & 0.52 \\
\hline Butyrate & $1.85^{\mathrm{a}}$ & 0.11 & $3.47^{b}$ & 0.14 & $6 \cdot 91^{\mathrm{c}}$ & 0.23 & $2 \cdot 82^{d}$ & 0.10 \\
\hline Ratio A:P & $6 \cdot 49^{\mathrm{a}}$ & 0.51 & $6.58^{\mathrm{a}}$ & 0.34 & $10 \cdot 49^{b}$ & 0.47 & $5 \cdot 56^{\mathrm{a}}$ & 0.27 \\
\hline \multicolumn{9}{|c|}{ Molar concentration $(\mathrm{mM})^{\star}$} \\
\hline Total & $14 \cdot 95^{\mathrm{a}}$ & 0.79 & $17 \cdot 71^{b}$ & 0.87 & $12 \cdot 98^{\mathrm{a}, \mathrm{c}}$ & 0.50 & $11 \cdot 16^{\mathrm{c}}$ & 0.49 \\
\hline Lactate & $3.37^{\mathrm{a}}$ & 0.13 & $2.59^{\mathrm{b}}$ & 0.09 & $2 \cdot 33^{\mathrm{c}}$ & 0.04 & $2 \cdot 28^{\mathrm{c}}$ & 0.01 \\
\hline \multicolumn{9}{|l|}{ Log CFU/g } \\
\hline Bacteroides & $6 \cdot 96^{\mathrm{a}}$ & 0.05 & $7 \cdot 87^{\mathrm{b}}$ & 0.08 & $7.34^{\mathrm{c}}$ & 0.02 & $8.53^{d}$ & 0.01 \\
\hline Bifidobacterium & $7 \cdot 55^{a}$ & 0.04 & $8 \cdot 12^{b}$ & 0.07 & $7 \cdot 16^{\mathrm{c}}$ & 0.09 & $7 \cdot 32^{c}$ & 0.03 \\
\hline Enterobacteriaceae & $9 \cdot 64^{\mathrm{a}}$ & 0.04 & $9.57^{\mathrm{a}}$ & 0.06 & $8 \cdot 56^{\mathrm{b}}$ & 0.03 & $8.25^{\mathrm{c}}$ & 0.03 \\
\hline Enterococcaceae & $7 \cdot 52^{\mathrm{a}}$ & 0.02 & $7 \cdot 63^{b}$ & 0.06 & $5 \cdot 61^{c}$ & 0.03 & $6 \cdot 95^{d}$ & 0.03 \\
\hline Weissella & $5 \cdot 12^{\mathrm{a}}$ & 0.05 & $4 \cdot 65^{\mathrm{b}}$ & 0.07 & $3.68^{c}$ & 0.03 & $3.90^{d}$ & 0.05 \\
\hline
\end{tabular}

A:P, acetic acid:propionic acid ratio; CFU, colony-forming units.

$\mathrm{a}, \mathrm{b}, \mathrm{c}, \mathrm{d}$ Mean values with unlike superscript letters are significantly different for a given variable between the different population groups $(P<0.05)$.

* Total SCFA is the sum (in $\mathrm{mm}$ ) of acetic, propionic and butyric acids. Molar proportions of acetic, propionic and butyric acids are expressed as a percentage of total SCFA. 
external carbon source added, were prepared. The $\mathrm{pH}$ values of the faecal batch cultures with bifidobacteria added decreased from $6 \cdot 11-6.68$ to $4.08-4.43$ from the start until $48 \mathrm{~h}$ of incubation, whereas the cultures with FOS attained lower $\mathrm{pH}$ values, which were between 3.68 and 3.91. Lactic acid and SCFA levels as well as microbial counts were determined at 5 and $48 \mathrm{~h}$ of incubation (see Supplementary data, available online). A comparison of the increments of the values obtained with respect to time 0 was made.

The repeated-measures ANOVA test showed significant differences in the levels of lactic acid, total SCFA and butyric acid and the acetic acid:propionic acid ratio as well as in the counts of Bacteroides, Enterobacteriaceae and Enterococcaceae depending on the incubation time of the faecal cultures. Significantly higher levels of lactic acid were found in cultures to which FOS were added with respect to the negative control $(P<0.05)$. No significant differences $(P>0.05)$ attributable to the treatments, i.e. FOS and bifidobacterial strains, were found among faecal cultures for the parameters analysed. In contrast, the statistical analysis revealed differences $(P<0.05)$ among cultures for all the parameters, except for Weissella, considered in the present study (lactic acid, SCFA and microbial population counts) depending on the human population group origin of the faecal samples. Since no differences among treatments were observed as evidenced by the previous statistical analysis, results obtained for all treatments (except for the negative control) for the different parameters (except for Weissella) were considered jointly (means and standard errors) and are presented in Fig. 1. During incubation, it was identified that two clearly different patterns in the time course of acetic and propionic acids were dependent on the human population group. In cultures from premature babies, the molar proportions of acetic acid decreased, whereas those of propionic acid increased during incubation. However, the opposite behavioural pattern was found for cultures from the remaining human groups, in which the molar proportions of acetic acid increased and the proportion of propionic acid decreased. Cultures from the group of breastfed infants exhibited the most pronounced decreases in the molar proportions of butyrate and the sharpest increases in the acetic acid:propionic acid ratio. Differences occurring in total SCFA and lactic acid levels among faecal cultures from the different human population groups were less pronounced. These results point to the human population group as the predominant factor, compared with the bifidobacteria or FOS added, in determining the profile of SCFA in in vitro faecal cultures from adults and newborns. With respect to the intestinal microbial groups analysed, the counts of Bacteroides showed more pronounced increases in the faecal cultures from formula-fed infants than in those from the other human groups. Lower increases of bifidobacteria were observed in the faecal cultures from breast-fed infants than in those from premature babies and adults. The faecal cultures from premature babies displayed the most pronounced decreases of Enterobacteriaceae during incubation, whereas the faecal cultures from adults exhibited more pronounced increases of Enterococcaceae than those from the other groups. The present results indicate that the human group, and therefore the basal microbiota, determines variations in the response of some microbial populations to the presence of some bifidobacterial strains or FOS in faecal cultures.

Finally, from the repeated-measures ANOVA test, an interaction between the human population group and the time of incubation was observed for the levels of lactic, acetic, propionic and butyric acids and the acetic acid:propionic acid ratio as well as for the counts of Bifidobacterium and Enterobacteriaceae attained in the faecal cultures. This statistical interaction points to an influence of the human group analysed over the progress of these parameters during incubation. Additionally, an interaction between the time of incubation and the addition of FOS was also found for the levels of lactic acid in the faecal cultures. This statistically translates the higher increase of lactic acid occurring at late stages of incubation ( $48 \mathrm{~h}$ ) in the faecal cultures with FOS added with respect to the other treatments assayed (bifidobacteria) (see Supplementary data, available online).

\section{Discussion}

Despite the high inter-individual variability found, the present results showed clear differences in SCFA levels and microbial groups in faecal slurries from the different human populations analysed after the stabilisation period. In a previous human study, we obtained lower levels of acetic and propionic acids in preterm babies during the first few days of life, when compared with full-term breast-fed infants, whereas no differences were found in their molar proportions; butyrate levels were scarce in both infant groups ${ }^{(5)}$. In contrast, in the present study, when comparing faecal slurries from both infant populations, after the stabilisation period in the in vitro model, we obtained clearly higher proportions of propionic and butyric acids in the premature and breast-fed baby samples, respectively. This and other differences in the SCFA profile found, although do not actually fit the initial composition in faeces, reflect a distinct metabolic activity of the intestinal microbiota from the human population groups analysed. The stabilised faecal samples from premature babies exhibited lower levels of Bacteroides and higher levels of Enterobacteriaceae, Enterococcaceae and Weissella than those from breast-fed infants, which was coincident in this case with that stated previously for faeces from both population groups ${ }^{(5)}$. Although a delayed gut colonisation by bifidobacteria has been reported in preterm newborns with respect to full-term breast-fed infants ${ }^{(5,21)}$, we found significantly more bifidobacteria in the faecal slurries from premature infants than in those from breast-fed infants, after the stabilisation period. In this respect, a more pronounced increase of bifidobacteria when these micro-organisms are at lower levels has been reported previously during the incubation of faecal cultures with prebiotics ${ }^{(22,23)}$

After the stabilisation step, faecal cultures were prepared with different bifidobacteria or FOS being added. Clear differences were found among the human population groups but not among the treatments (different strains or FOS). In addition, the time course of most parameters during incubation of the faecal cultures was also dependent on the 
(a)

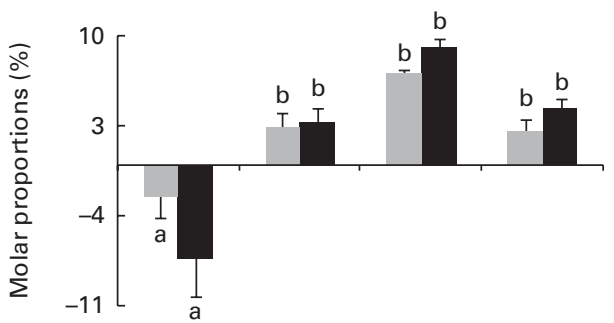

(c)
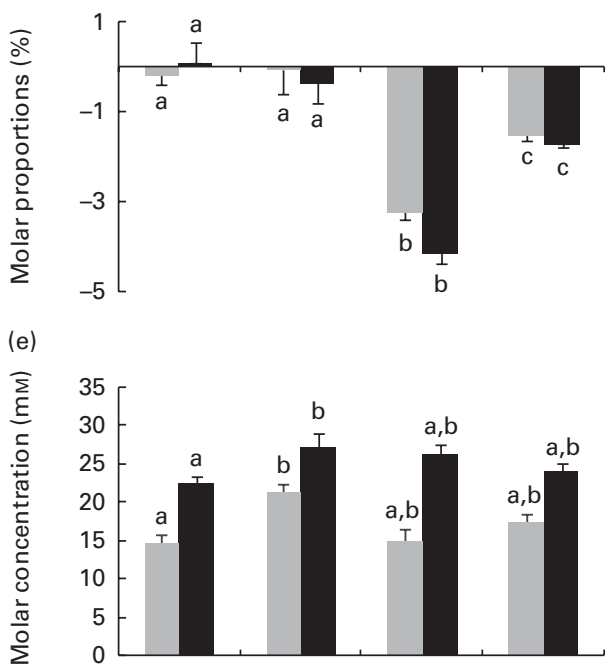

(g)

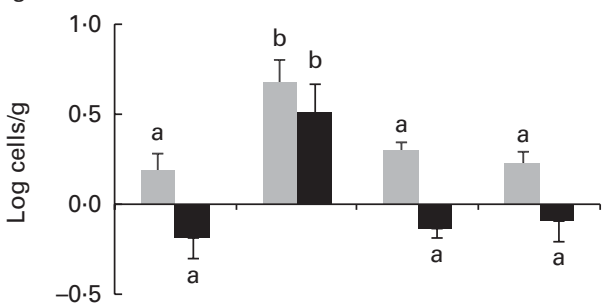

(i)

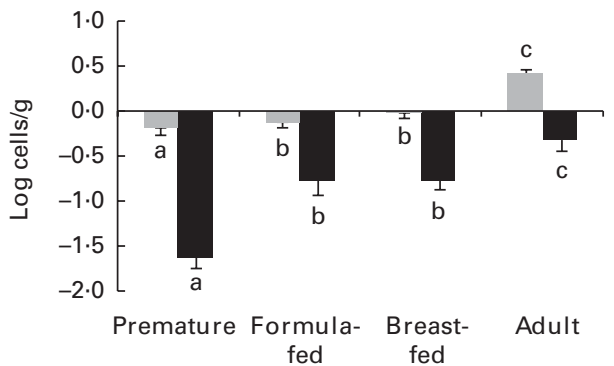

(b)

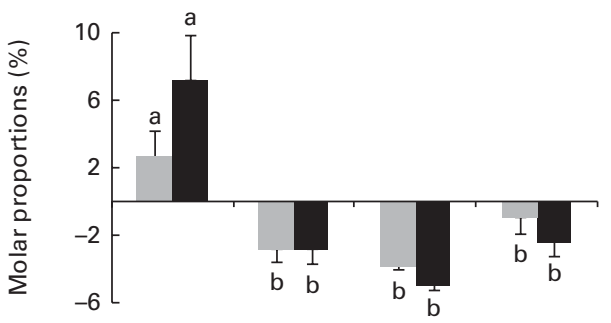

(d)

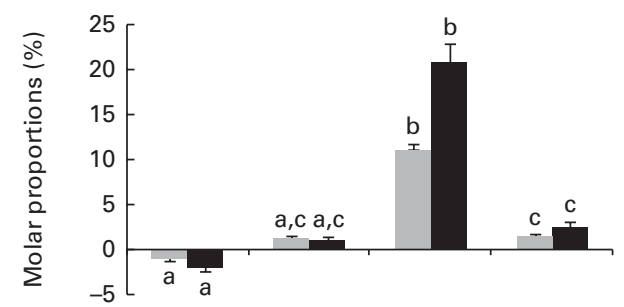

(f)

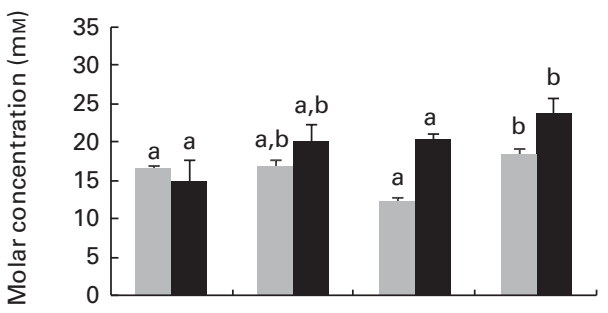

(h)

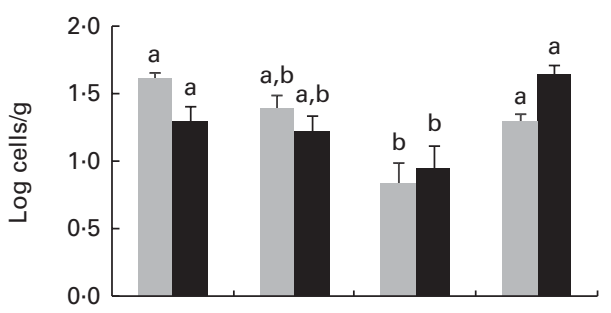

(j)

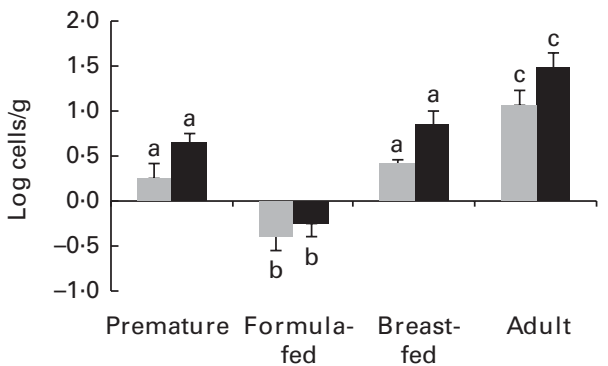

Fig. 1. Increments at $5 \mathrm{~h} \mathrm{( \square )}$ and $48 \mathrm{~h}(\square)$ of incubation with respect to time 0 of (a) acetate, (b) propionate and (c) butyrate molar proportions (expressed as the percentage of total SCFA), (d) acetic:propionic acid ratio, (e) total SCFA, (f) lactate, and (g) Bacteroides, (h) Bifidobacterium, (i) Enterobacteriaceae and (j) Enterococcaceae population levels determined by quantitative PCR in $\mathrm{pH}$-free batch cultures of three faecal mixtures corresponding to breast-fed infants, formula-fed infants, preterm babies (numerical data from this last group were taken from Arboleya et al. ${ }^{(16)}$ ) and adults with bifidobacteria and fructo-oligosaccharides added. Values are means, with their standard errors represented by vertical bars. ${ }^{a, b, c}$ Mean values with unlike letters are significantly different among the population groups $(P<0.05)$ 
human group origin of the faecal samples. Thus, the faecal cultures from breast-fed infants displayed the most pronounced decreases in the molar proportions of butyrate and the most pronounced increases in the acetic acid:propionic acid ratio among the four population groups. The molar proportions of acetic acid progressively reduced and those of propionic acid progressively increased in the faecal cultures from premature babies, whereas the opposite was observed in the faecal cultures from the remaining human groups, for which we found an increase in the molar proportions of acetic acid and a reduction in those of propionic acid during incubation. These results point to the initial composition of the microbiota as a predominant factor, compared with bifidobacteria or FOS added, in determining the concentration of SCFA in in vitro faecal cultures. Regarding the microbial populations, we observed variations among the human groups in the response of Bacteroides, Bifidobacterium, Enterobacteriaceae and Enterococcaceae to FOS and Bifidobacterium strains added to the faecal cultures, whereas no significant differences were found for Weissella. This shows that the background microbiota play a crucial role in determining the response of the intestinal microbiota to bifidobacteria or FOS added.

Finally, although differences in the production of lactic acid were found among faecal cultures of the different human groups, this acid displayed more pronounced increases at late stages of incubation in the faecal cultures with FOS added than in those with bifidobacteria added, thus highlighting a differential effect of the prebiotic substrate occurring at prolonged times of incubation.

Previous studies have indicated a high inter-individual variability in the response to probiotics ${ }^{(14,15)}$. In the present study, we have shown, using an in vitro model, a strong dependence of the microbiota-modulating ability of bifidobacteria and FOS on the background microbiota of the studied human groups. However, most of the currently available probiotic strains and prebiotic products target the general population and have not been rationally selected on the basis of the needs of, and their effects on, specific human populations $^{(24)}$. The present study exhibits important differences in the response to bifidobacterial strains or FOS in the faecal cultures of the intestinal microbiota from different human groups. This highlights the need to design probiotics targeting specific human population groups and to avoid extrapolation from a given human group to others.

\section{Supplementary material}

To view supplementary material for this article, please visit http://dx.doi.org/10.1017/S0007114513001487

\section{Acknowledgements}

The present study was financially supported by European Union FEDER funds and by the Spanish Ministry of Economy and Competitiveness under the project AGL2010-16525 and by CSIC through the project PIE201370E19. S. A. was funded by a predoctoral JAE fellowship from CSIC, Spain.
María Fernández-García, Ana M. Hernández-Barranco and Isabel Cuesta are greatly appreciated for their excellent technical assistance.

S. A. conducted the experiments, analysed the results and drafted the paper. N. S. conducted the experiments and drafted the paper. G. S. and N. F. provided faecal samples of the babies and drafted the paper. M. G. and C. G. d. 1. R.-G. designed and supervised the study along with drafting the manuscript. The authors have no conflicts of interest.

\section{References}

1. Penders J, Thijs C, Vink C, et al. (2006) Factors influencing the composition of the intestinal microbiota in early infancy. Pediatrics 118, 511-521.

2. Harmsen HJM, Wildeboer-Veloo ACM, Raangs GC, et al. (2000) Analysis of intestinal flora development in breastfed and formula-fed infants by using molecular identification and detection methods. I Pediatr Gastroenterol Nutr 30, 61-67.

3. Bezirtzoglou E, Tsiotsias A \& Welling GW (2011) Microbiota profile in feces of breast-and formula-fed newborns by using fluorescence in situ hybridization (FISH). Anaerobe 17, 478-482.

4. Poroyko V, Morowitz M, Bell T, et al. (2011) Diet creates metabolic niches in the "immature gut" that shape microbial communities. Nutr Hosp 26, 1283-1295.

5. Arboleya S, Binetti A, Salazar N, et al. (2012) Establishment and development of intestinal microbiota in preterm neonates. FEMS Microbiol Ecol 79, 763-772.

6. Arboleya S, Solís G, Fernández N, et al. (2012) Facultative to strict anaerobes ratio in preterm infant microbiota: a target for intervention? Gut Microb 3, 583-588.

7. Amarri S, Benatti F, Callegari ML, et al. (2006) Changes of gut microbiota and immune markers during the complementary feeding period in healthy breast-fed infants. Pediatr Gastroenterol Nutr 42, 488-495.

8. FAO/WHO (2006) Probiotics in Food. Health and Nutritional Properties and Guidelines for Evaluation. FAO Food and Nutrition Paper no. 85. FAO/WHO. ftp://ftp.fao.org/ docrep/fao/009/a0512e/a0512e00.pdf

9. Floch MH, Walker WA, Madsen K, et al. (2011) Recommendations for probiotic use - 2011 update. J Clin Gastroenterol 45, S168-S171.

10. Sanz Y (2011) Gut microbiota and probiotics in maternal and infant health. Am J Clin Nutr 94, 2000S-2005S.

11. Grzeskowiak L, Isolauri E, Salminen S, et al. (2011) Manufacturing process influences properties of probiotic bacteria. Br J Nutr 105, 887-894

12. Kalliomaki M, Salminen S, Arvilommi H, et al. (2001) Probiotics in primary prevention of atopic disease: a randomised placebo-controlled trial. Lancet 357, 1076-1079.

13. Kopp MV, Hennemuth I, Heinzmann A, et al. (2008) Randomized, double-blind, placebo controlled trial of probiotics for primary prevention: no clinical effects of Lactobacillus GG supplementation. Pediatrics 121, 850-856.

14. Van Baarlen P, Troost F, van der Meer C, et al. (2011) Human mucosal in vivo transcriptome responses to three lactobacilli indicate how probiotics may modulate human cellular pathways. Proc Natl Acad Sci U S A 108, 4562-4569.

15. Grzeskowiak L, Grönlund MM, Beckmann C, et al. (2012) The impact of perinatal probiotic intervention on gut microbiota: double-blind placebo-controlled trials in Finland and Germany. Anaerobe 18, 7-13. 
16. Arboleya S, Salazar N, Solís G, et al. (2013) Assessment of intestinal microbiota modulation ability of Bifidobacterium strains in in vitro fecal batch cultures from preterm neonates. Anaerobe 19, 9-16.

17. Garrigues C, Stuer-Lauridsen B, Johansen E, et al. (2005) Characterisation of Bifidobacterium animalis subsp. lactis Bb12 and other probiotic bacteria using genomics, transcriptomics and proteomics. Aust J Dairy Technol 60, 84-92.

18. Arboleya S, Ruas-Madiedo P, Margolles A, et al. (2011) Characterization and in vitro properties of potentially probiotic Bifidobacterium strains isolated from breast-milk. Int J Food Microbiol 149, 28-36.

19. Young JPW, Downer HL, Eardly BD, et al. (1991) Phylogeny of the phototrophic Rhizobium strain BTAil by polymerase chain reaction-based sequencing of a $16 \mathrm{~S}$ rRNA gene segment. J Bacteriol 173, 2271-2277.

20. Salazar N, Prieto A, Leal JA, et al. (2009) Production of exopolysaccharides by Lactobacillus and Bifidobacterium strains of human origin, and metabolic activity of the producing bacteria in milk. J Dairy Sci 92, 4158-4168.

21. Butel MJ, Suau A, Campeotto F, et al. (2007) Conditions of bifidobacterial colonization in preterm infants: a prospective analysis. J Pediatr Gastroenterol Nutr 44, $577-582$.

22. De Preter V, Vanhoutte T, Huys G, et al. (2008) Baseline microbiota activity and influence responses to prebiotic initial bifidobacteria counts dosing in healthy subjects. Aliment Pharmacol Ther 27, 504-513.

23. Cardelle-Cobas A, Fernández M, Salazar N, et al. (2009) Bifidogenic effect and stimulation of short chain fatty acid production in human faecal slurry cultures by oligosaccharides derived from lactose and lactulose. J Dairy Res 76, 317-325.

24. Arboleya S, González S, Salazar N, et al. (2012) Development of probiotic products for nutritional requirements of specific human populations. Eng Life Sci 12, 368-376. 